\title{
Diseño y análisis de productos y espacios con técnicas de prototipado virtual interactivo
}

\section{Begoña Sáiz Mauleón ${ }^{a}$, Eugenio Ivorra Martínez ${ }^{b}$, Pedro Verdejo Gimeno ${ }^{c}$, Juan J. Cisneros Vivóa} ${ }^{a}$ Departamento de Expresión Gráfica Arquitectónica, Escuela Técnica Superior de Ingeniería del Diseño, Universitat Politècnica de València, bsaizma@ega.upv.es, b Departamento de Ingeniería de Sistemas y Automática, Escuela Técnica Superior de Ingeniería del Diseño, Universitat Politècnica de València, euivmar@i3b.upv.es, ${ }^{\mathbf{c} D e p a r t a m e n t o ~}$ de Proyectos, Teoría y Técnica del Diseño y Arquitectura, Universitat CEU Cardenal Herrera, pverdejo@uchceu.es

\begin{abstract}
This work shows how the use of immersive visualization techniques can influence the creative process, improving the projected ideas and making the analysis of the products and spaces designed easier. The innovative process of the course "Advanced Visualizations. Virtual reality applied to the design of products" taught in the Master's Degree in Design Engineering, ETSID-UPV, where the students think, design and experiment with three-dimensional models visualized on twodimensional media, models and immersive virtual prototypes. The work is shown from the motivation, the surprise and the approach to the most tangible reality and a set of actions derived from that approach that allows the student to see his project come real. The evolution of technology and its implementation at the university allows, to improve teaching with tools that are already available to students, making the teaching experience the maximum approach to the career that will soon come to them.
\end{abstract}

Keywords: HTC Vive, CAVE, Virtual Reality, Product Design, Teaching innovation

\section{Resumen}

El trabajo muestra cómo el empleo de técnicas de visualización inmersiva influyen en el proceso creativo, mejorando las ideas proyectadas y facilitando un análisis de los productos y espacios ideados. Se plasma el proceso innovador de la asignatura "Visualizaciones avanzadas. Realidad virtual aplicada al diseño de productos" impartida en el Máster Universitario de Ingeniería del Diseño, de la ETSID-UPV, en donde el alumnado idea, diseña y experimenta con modelos tridimensionales, visualizados en soportes bidimensionales y maquetas y prototipos virtuales inmersivos. Se muestra el trabajo desde la motivación, la sorpresa, la aproximación a la realidad más tangible y un conjunto de acciones derivadas de esa aproximación que posibilita al estudiante ver su proyecto hecho realidad. La evolución de la tecnología y su implementación en la universidad permite, a su vez, implementar la docencia con herramientas que ya están al alcance del alumnado, convirtiendo la experiencia docente en la máxima aproximación a la realidad laboral que en breve les espera.

Palabras clave: HTC Vive, CAVE, Virtual Reality, Product Design, Innovación docente 


\section{Introducción}

La comunicación se basa en la experiencia y resultados obtenidos en la asignatura "Visualizaciones Avanzadas. Realidad virtual aplicada al diseño de productos" impartida desde el curso 2011-2012 en el Máster Universitario en Ingeniería del Diseño, en la Escuela Técnica Superior de Ingeniería del Diseño (ETSID), de la Universitat Politècnica de Valéncia (UPV). Así mismo, se plasman experiencias derivadas de Trabajos Finales de Máster (TFM) de estudiantes que han optado por ampliar y dar continuidad a la realidad virtual inmersiva como herramienta profesional en el diseño de productos y espacios.

Desde el inicio de su andadura en la asignatura se ha trabajado con dos técnicas distintas. Desde el curso 2011-2012 la tecnología empleada fue el Cave Automatic Virtual Environment (CAVE) y a partir del curso 2016-2017 hasta la actualidad se han empleado las gafas HTC Vive como herramienta de trabajo en la estrategia docente de aprendizaje activo y colaborativo. Así mismo, durante el curso 2014-2015 se testó con el alumnado, a modo de ensayo experimental de cara a su futuro uso docente, las Oculus Rift, siendo finalmente descartadas, como veremos a lo largo del trabajo.

La asignatura plantea una primera parte de trabajo individual en donde las primeras ideas esbozadas son corregidas de forma colectiva, para posteriormente pasar a trabajar en equipo, formando grupos por afinidad formal, fusionando y mejorando las propuestas en base a los requerimientos planteados y pasando, a partir de este momento, a digitalizar las propuestas para proceder a su estudio mediante su inmersión en la realidad virtual.

Son numerosos los estudios que muestran cómo el empleo de la realidad virtual interviene en los procesos creativos (Rubio-Tamayo, 2016) y potencia la habilidad espacial mejorando de forma inconsciente nuestra capacidad de comprensión tridimensional, (Molina, 2018).

El manejo de entornos virtuales en los estudios de ingeniería (Richert, 2016) va más allá de potenciar el aprendizaje de las materias, e incluso de motivar. El compromiso adquirido de preparar al futuro egresado para los trabajos que la industria 4.0. requiere pasa por darle una visión amplia del sentido de esta herramienta y su valía profesional (Salah, 2019).

Es por ello que los estudios de ingeniería deben ofrecer al estudiante la posibilidad de conocer y aprender cómo los entornos virtuales pueden ayudarle (Vergara, 2017), no solo en los procesos de definición del producto, sino también en futuras habilidades a desarrollar y que ya formará de aprendizaje continuo que se le debe inculcar desde las aulas.

\section{Objetivos}

En el marco del diseño de producto la asignatura tiene una estrategia fundamentalmente motivadora, con el objetivo de trasmitir la importancia del aprendizaje constante, como valor añadido en la implementación de novedades para nuevos productos del mercado. Este valor añadido debe ser el elemento diferenciador de la competencia. Empleando todos los pasos de un proceso de diseño (estudio de campo, análisis previos, ideación, bocetado, conceptualización, dibujo por ordenador y visualización del producto) el alumnado entiende cómo los avances de la técnica, en este caso la realidad virtual inmersiva, posibilita una visualización real del producto desde un prototipo virtual, marcando una clara diferencia de la visualización $3 \mathrm{~d}$ sobre un soporte bidimensional, como es una pantalla. 
Los objetivos generales y concretos de la asignatura son:

\subsection{Objetivos generales}

- Motivar al alumnado empleando herramientas de diseño innovadoras.

- Innovar mediante el aprendizaje de tecnologías aplicadas al proceso de diseño.

- Inculcar el sentido del aprendizaje continuo.

- Promover el trabajo colaborativo mediante correcciones grupales.

- Aprender a resolver conflictos dentro del trabajo en equipo.

- Trabajar desde la transversalidad del conjunto de técnicas empleadas en el proceso de diseño.

- Ofrecer al alumnado herramientas de valor añadido para la defensa de su proyecto.

- Valorar las técnicas empleadas en búsqueda permanente de sistemas o productos que permitan mejorar y motivar al alumnado durante su proceso de aprendizaje.

\subsection{Objetivos específicos}

- Diseñar un producto de uso colectivo atendiendo a la diversidad funcional del usuario.

- Aplicar todas las herramientas aprendidas en un proceso de diseño.

- Modelar para visualizar en tiempo real.

- Evidenciar las diferencias de hacer un análisis del diseño mediante soporte bidimensional (monitor) y soporte tridimensional (prototipo virtual inmersivo).

- Analizar las maquetas virtuales de trabajo desde la inmersión e interacción.

- Rediseñar las maquetas virtuales y definir el prototipo virtual del producto final, atendiendo al diseño de detalle.

- Contextualizar y naturalizar el producto de cara a una presentación final a un comprador/empresa.

\section{Desarrollo de la innovación}

La experiencia creativa, a la hora de desarrollar un nuevo producto, no solo se aplica en la ideación sino también al número de herramientas y modo en el que el diseñador es capaz de emplearlas, tanto por su aprendizaje en el aula como por su propio desarrollo subjetivo. La realidad virtual, empleada fundamentalmente como herramienta de análisis formal y de escala, no se trabaja aisladamente del dibujo $\mathrm{u}$ otras herramientas, sino que hay un concepto integral en todo el planteamiento de la asignatura, de modo que, partiendo de la propuesta inicial, y tras un estudio de mercado el alumnado trabaja con bocetos, maquetas, collages, hasta modelizar la propuesta para su análisis mediante la maqueta virtual. 

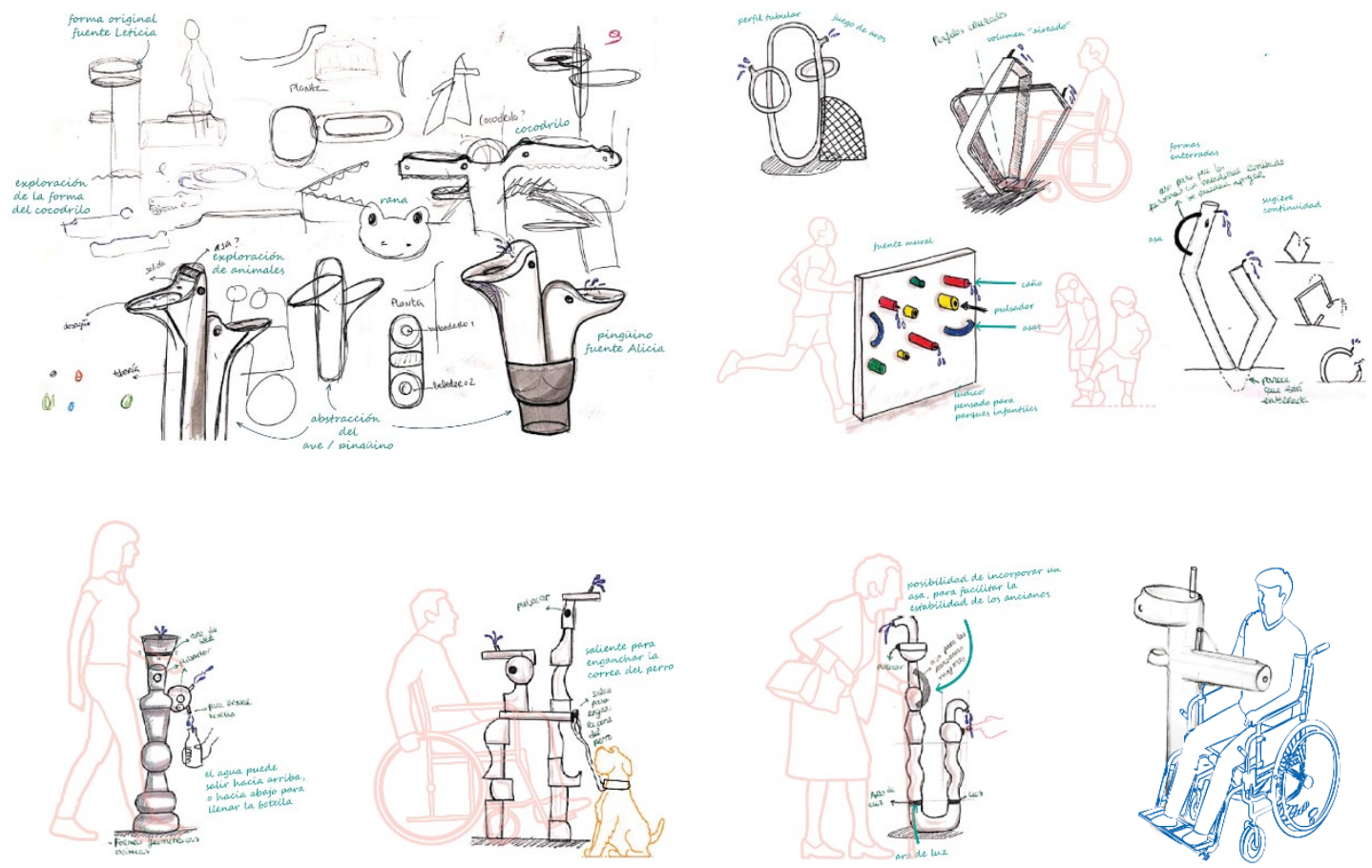

Fig. 1 Bocetos iniciales (fila superior) y evolución del diseño (fila inferior) de una fuente bebedero con criterio de diversidad funcional. Alumnas Leticia Lucia Campos y Alicia Alonso Gil. Curso 2019-2020

Desde los cursos 2011-2012 hasta 2015-2016 el alumnado de la asignatura estuvo empleando la CAVE de la UPV, gestionada por el Área de Sistemas de la Información y Comunicaciones (ASIC), para visualizar sus maquetas virtuales de modo inmersivo. El tamaño prefefinido de esta CAVE es de de 2,35 x 2,5 x 2,5m y el análisis de los productos y espacios ideados ofreció al alumnado una nueva experiencia de interpretación de sus propuestas gracias a la inmersión, interacción y observación a escala real (Sáiz, 2010). En ese momento el empleo de esta tecnología en el marco docente no estaba tan extendido como ahora. Los resultados obtenidos y la acogida de esa nueva herramienta, no solo en esta asignatura, sino en otras del propio máster (Val, 2015) marcaron un punto de inflexión a partir del cual el análisis y la evaluación del propio trabajo adquirió una nueva dimensión gracias a una mejor interpretación de las escalas del producto en sí mismo, con el usuario y con el entorno.
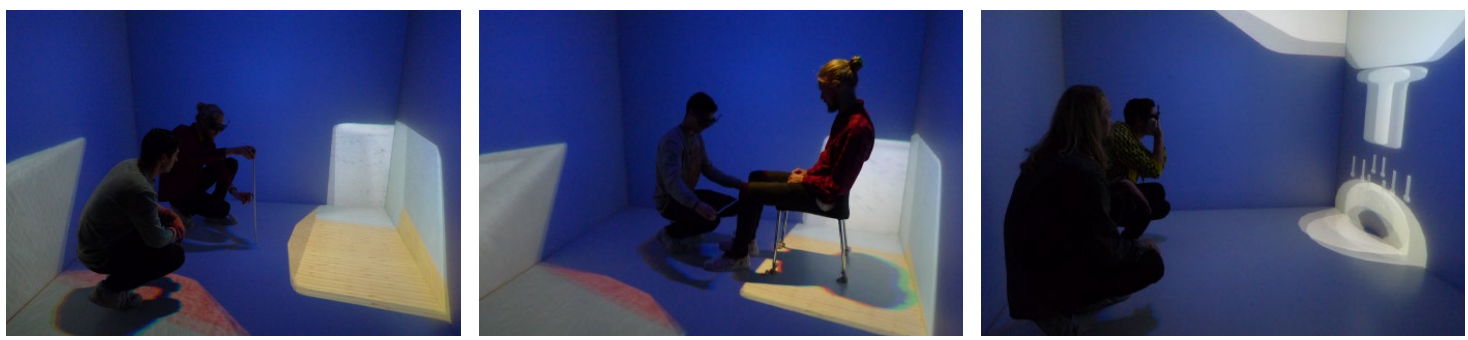

Fig. 2 Los estudiantes Javier Gómez y Anton Wallner del curso 2015-2016, analizando la escala de su producto, la sensación espacial de la usabilidad y el diseño en detalle del anclaje del elemento de descanso.

(c)) BY-NC-ND 2020, Universitat Politècnica de València

Congreso In-Red (2020) 
Las imágenes de la figura 2 muestran una de las ventajas de la CAVE, que era la posibilidad del análisis dual, en donde ambos estudiantes podían compartir a la vez el momento del análisis, que no de la visualización e interacción. Pero aún así, este detalle era un momento interesante en donde el profesorado observaba la evolución de los estudiantes, no solo desde la perspectiva del análisis y desarrollo del diseño sino también desde la relación dentro del grupo de trabajo. El intercambio fácil de las gafas estereocópicas y su propia corporeidad dentro de la escena proyectada en el espacio de la CAVE, hacía muy real la interacción con el objeto.

En el caso del análisis espacial, la validación de un espacio como herramienta de trabajo se muestra en las siguientes imágenes, en donde gracias al empleo de la CAVE se pudo testear en el prototipo virtual la sensación espacial de las distancias en espacios reducidos de trabajo (Bogard, 2015) o en el diseño de una cocina doméstica (Rodriguez, 2012).
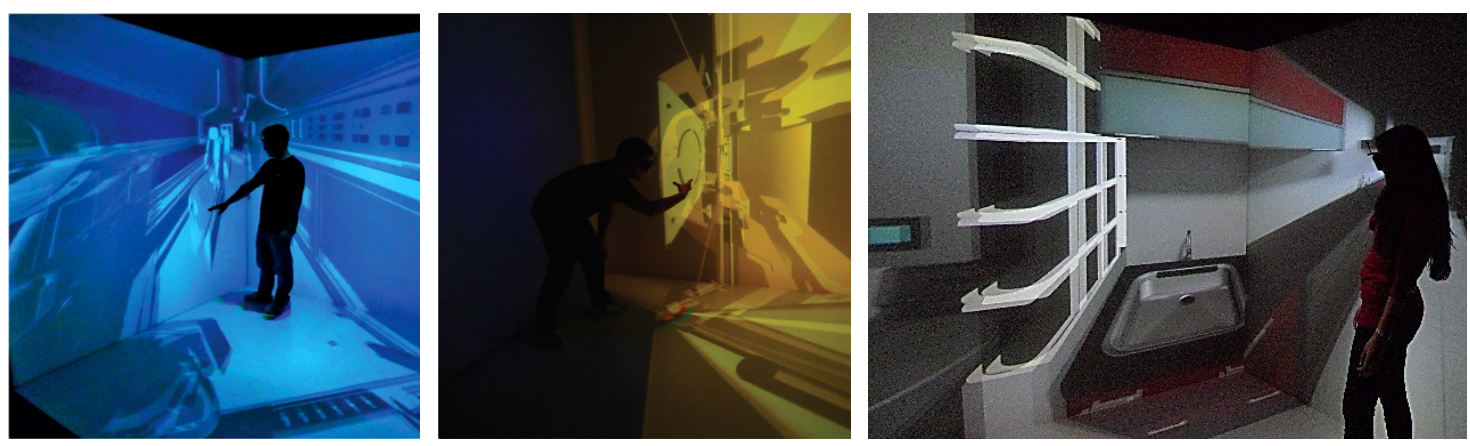

Fig. 3 Javier Bogard Domingo analizando el espacio de trabajo de un tren automatizado para su TFM (dos primeras imágenes). Andrea Rodríguez Chicote analizando la distribución espacial para su TFM (tercera imagen).

La CAVE presentaba un inconveniente importante derivado de su reducido tamaño. El hecho de no poderse desplazar por la escena más allá del espacio generado entre las cuatro paredes reducía muchos las expectativas de usabilidad en escenarios grandes.

A pesar de las ventajas anteriormente nombradas se valoró trabajar con otros dispositivos de visualización inmersiva, y durante el curso 2014-2015 se combinó en empleo de la CAVE con la experimentación con las Oculus Rift, a fin de comprobar al amlio campo de visión que prometían y la posibilidad de desplazarse en un espacio ligeramente mayor que la CAVE. El resultado no fue el esperado, generando en el $98 \%$ del alumnado una sensación desagradable de mareo y desubicación. Efectivamente, el campo de visión era de $360^{\circ}$, tal y como se prometía, pero la gestión de las escenas desde el teclado no permitía, ni siquiera, el desplazamiento del que se gozaba en la CAVE y el hecho de mover la escena sin la posibilidad de desplazar el cuerpo generaba una pérdida del equilibrio excesiva para el uso que de las gafas se exigía. Nunca se había experimentado con el alumnado esa sensación de mareo y malestar tras un breve uso de la visualización inmersiva.

En las imágenes de la figura 4 se observa cómo el alumnado efectivamente puede mirar en todas las direcciones, pero el hecho de gestionar la escena desde el teclado impedía ese mayor desplazamiento que se buscaba desde la CAVE. Así mismo, se aprecia como el alumnado se tenía que agarrar a la mesa debído a la pérdida del equilibrio que se generaba con el desplazamiento dentro de la escena. Afortunadamente los nuevos modelos de las Oculus han resuelto ese problema, pero en ese momento no se consideró una herramienta optima para intercambiarla por la CAVE. 

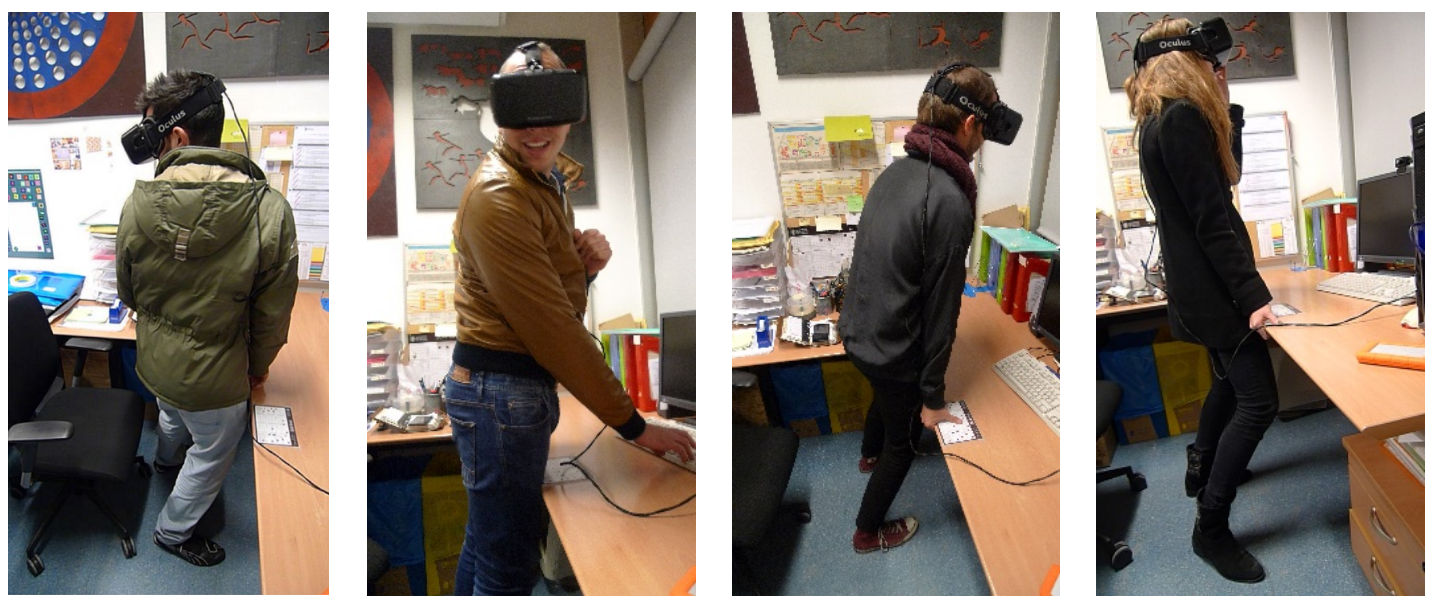

Fig. 4 Alumnado del curso 2014-2015 testando la Oculus Rift. Puede apreciase la gestión de movimientos por la escena desde el teclado y cómo se agarran a la mesa por la sensación de mareo y falta de estabilidad.

Fue a partir del año 2016-2017 y hasta la actualidad que se empezó a trabajar con las gafas de realidad virtual HTC Vive. La primera experiencia fue prometedora. Empleando una misma escena trabajada en la CAVE la trasladamos a las gafas en un escenario mucho mayor obteniendo una sensación de amplitud y libertad desconocida hasta el momento en el análisis virtual inmersivo. En la primera de las imágenes del conjunto inferior se obseva la escena proyectada en la CAVE en una superficie de $6,25 \mathrm{~m}^{2}$, el donde el estudiante puede apenas moverse alrededor del elemento de descanso. En la segunda imagen se observa el lugar de trabajo con las HTV Vive, de $20 \mathrm{~m}^{2}$ aproximadamente, en donde no solo hay posibilidad de moverse con libertad alrededor del elemento, sino que además la escena se puede desplazar, posibilitando la observación del elemento desde una distancia lejana y valorando de forma amplia la contextualización de los elementos dentro de una escena mucho mayor. En la tercera imagen se muestra la visualización a través de las HTC Vive con la rejilla azul que delimita el espacio de trabajo predefinido.
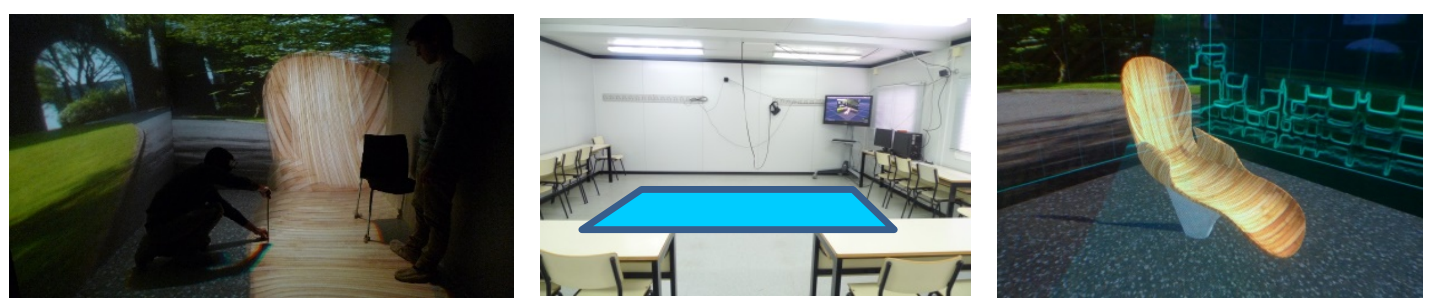

Fig. 5 Elemento de descanso ideado por los alumnos Gonzalo Rafael Acosta Zazueta y Pablo Argudo Torralba, del curso 2015 2016. En la primera imagen ambos estudiantes analizan el elemento desde la CAVE. En la segunda imagen se observa el espacio de trabajo con las HTC Vive, de unos $20 \mathrm{~m}^{2}$ y a la derecha la visualización inmersiva con las HTC Vive puestas.

A partir de este momento las mejoras en los trabajos de los estudiantes han sido notables. Sigue llamando la atención, como ya ocurría con la CAVE, el asombro que muestran los estudiantes cuando visualizan por primera vez su producto en el entorno inmersivo. A pesar de que llevan varias semanas modelandolo en $3 \mathrm{~d}$, se sorprenden de ver su propio elemento, dejando entrever que la percepción tridimensional imaginada al ver un modelo $3 \mathrm{~d}$ en la pantalla dista bastante en su imaginación de cómo el objeto es en realidad. Sin apenas esfuerzo el alumnado empieza a relatar posibles fallos o mejoras susceptibles llevar al diseño, haciendo de esa mirada inmersiva un traslado inmediato a la realidad conocida, para pasar seguidamente a

(c) B EY-NC-ND 2020, Universitat Politècnica de València

Congreso In-Red (2020) 
realizar una corrección observando y analizando en detalle el producto y mostrando al profesorado su propia evolución y madurez frente al trabajo realizado.

Como es de entender, en este caso lo que se observa desde fuera es lo que se muestra en el conjunto de imágenes de la figura 6. A diferencia de la CAVE nuestro cuerpo no está presente en la propia escena, sino que son los mandos que manejamos, y que aparecen dentro de la escena, los que nos vinculan espacialmente a la misma.
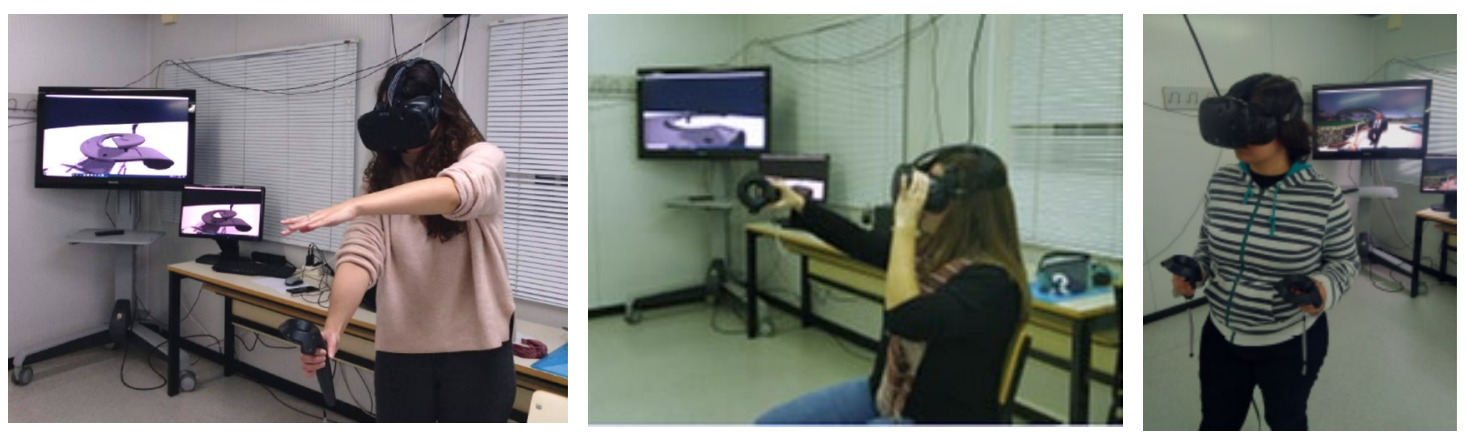

Fig. 6 Beatriz Martín Herrero, Cristina Redondo García y Silvia Vecino Mantilla, por este orden y del curso 2019-2020 interactuando con sus prototipos virtuales en el proceso de análisis final.

\section{Resultados}

Los resultados de la experiencia de innovación docente con la inmersión de la tecnología descrita, se han podido contrastar gracias a una experiencia anterior llevada a cabo en la asignatura del mismo máster "Diseño y Espacio Público" (Val, 2015) sobre su experiencia en entornos virtuales empleando la CAVE, y se compara con nuestra experiencia en la asignatura "Visualizaciones avanzadas. Realidad virtual aplicada al diseño de productos" con el empleo de las gafas HTV Vive.

\subsection{Descripción de la muestra en el estudio}

Se han registrado un total de 39 nuevas encuestas anónimas (cursos 2016-2017 hasta 2019-2020) para la evaluación de la metodología de realidad virtual basada en gafas HTC que se añaden a las 50 realizadas en cursos anteriores (Val, 2015) para la evaluación del uso de la realidad virtual con CAVE. El nuevo alumnado encuestado tiene una edad media de 26 años con una desviación típica de 6 . Además, se distribuyen en un $46 \%$ de hombres y un $54 \%$ de mujeres.

En la evaluación de metodologías basadas en realidad virtual está comprobado (Boot, 2011) que la experiencia de los sujetos con los videojuegos es una variable significativa. El número de horas que el alumnado juega a videojuegos a la semana y los dispositivos que utilizan puede observarse en la figura 7 . 


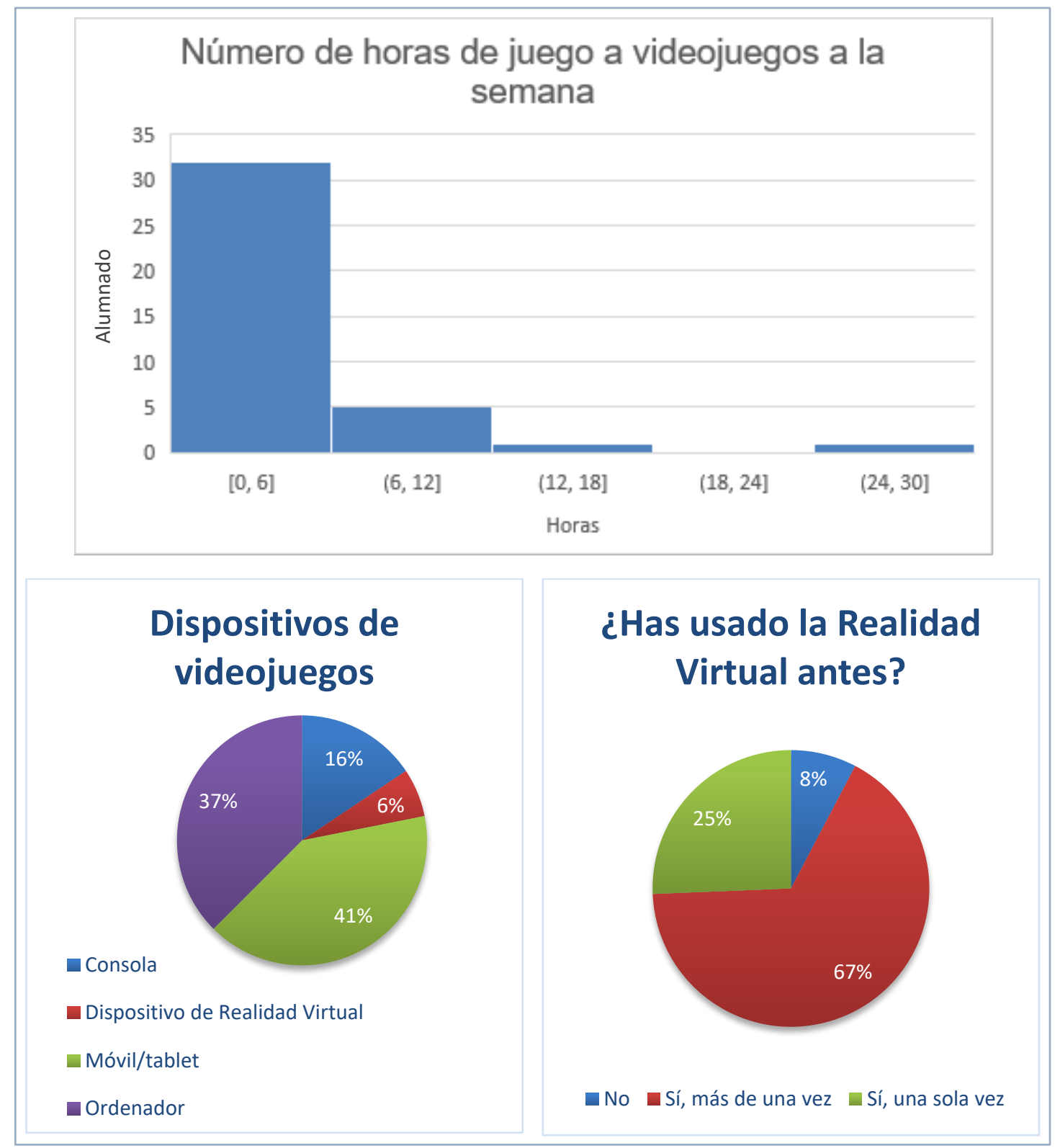

Fig. 7 Resultados de las encuestas acerca de la descripción de la experiencia en videojuegos y conocimiento de la RV

De acuerdo a la figura 7, la mayoría de gente juega menos de 6 horas a la semana, principalmente al móvil (41\%) y al ordenador (37\%). Un resultado significativo extraído de las encuestas es que el $92 \%$ del alumnado había empleado con anterioridad un dispositivo de RV y un $67 \%$ más de una vez. Este conocimiento previo de la tecnología permite una mejor integración y utilización con fines docentes.

\subsection{Comparativa entre tecnologías de RV}

Para esta comparativa se han empleado los resultados publicados por la profesora Val (Val, 2015) al utilizar una CAVE frente a los obtenidos en la asignatura "Visualizaciones Avanzadas. Realidad virtual aplicada al diseño de productos" empleando un dispositivo de realidad virtual portable (HTC). 
Al comparar la percepción de la tecnología se puede comprobar que nadie percibe las HTC como una tecnología complicada y remarcan su carácter innovador y estimulante, (Figura 8).

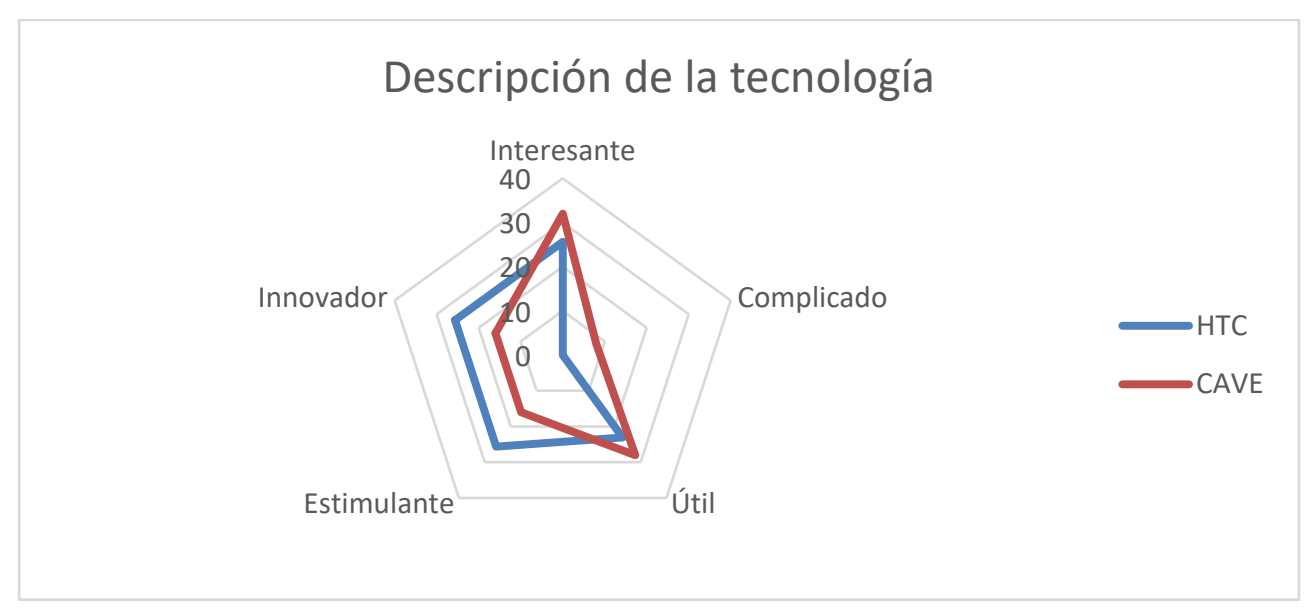

Fig. 8 Resultado de la pregunta: ¿Cómo describirías el uso de la tecnología?

En la siguiente figura (Fig. 9) el aspecto más relevante del uso de la realidad virtual es la visualización en contexto (48\%) al utilizar las HTC, suponiendo un incremento del 18\% frente a la utilización de una CAVE. Esto se debe a que el uso de las gafas permite una mayor sensación de inmersión que permite apreciar mejor las dimensiones y el contexto. Estos resultados son coherentes con los obtenidos en la adecuación de su utilización (Fig. 10) donde se aprecia un incremento del 32\% al 56\% al cambiar a las gafas.

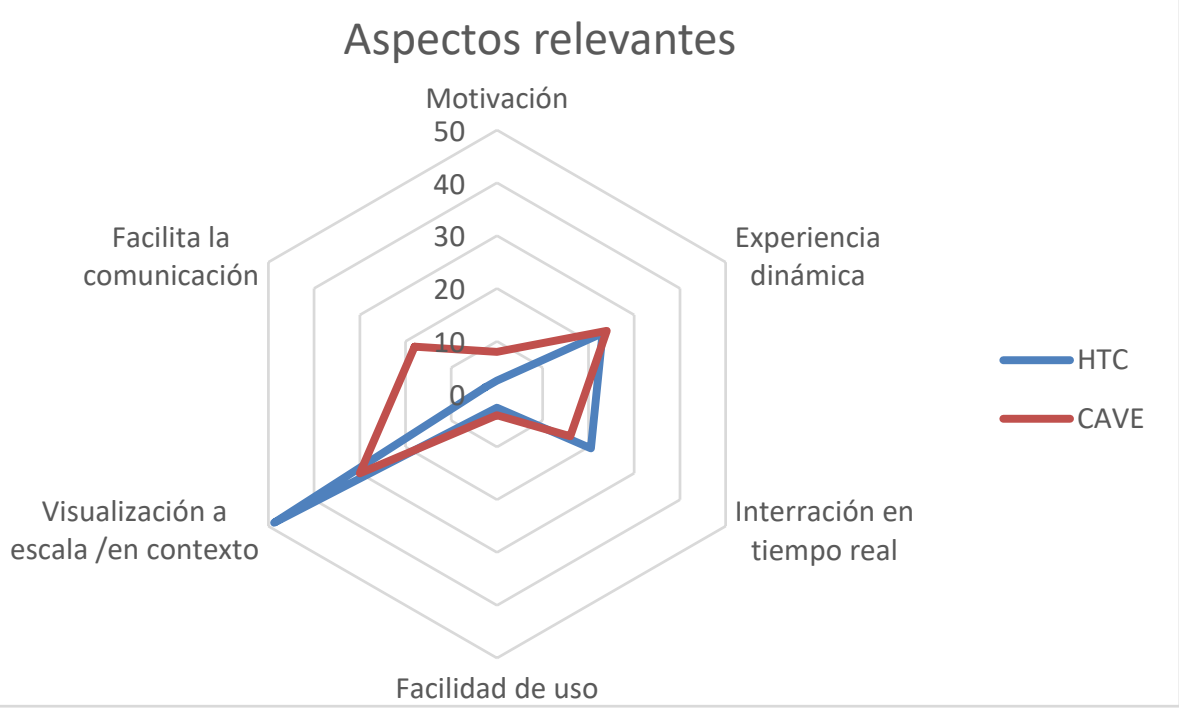

Fig. 9 Resultado de la pregunta: ¿Destaca los aspectos más relevantes del uso de RV? 


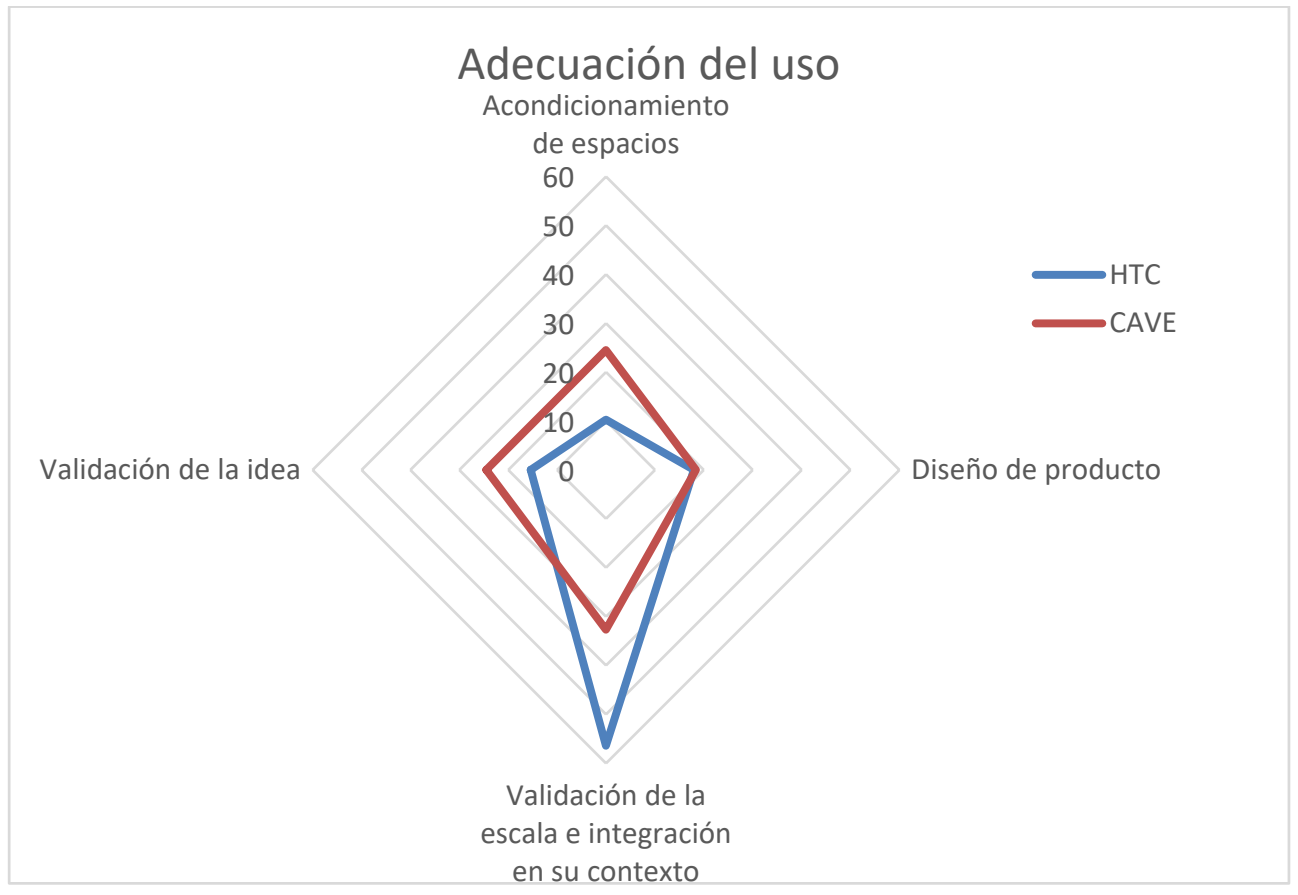

Fig. 10 Resultado de la pregunta: ¿Para qué consideras más adecuado el uso de la RV?
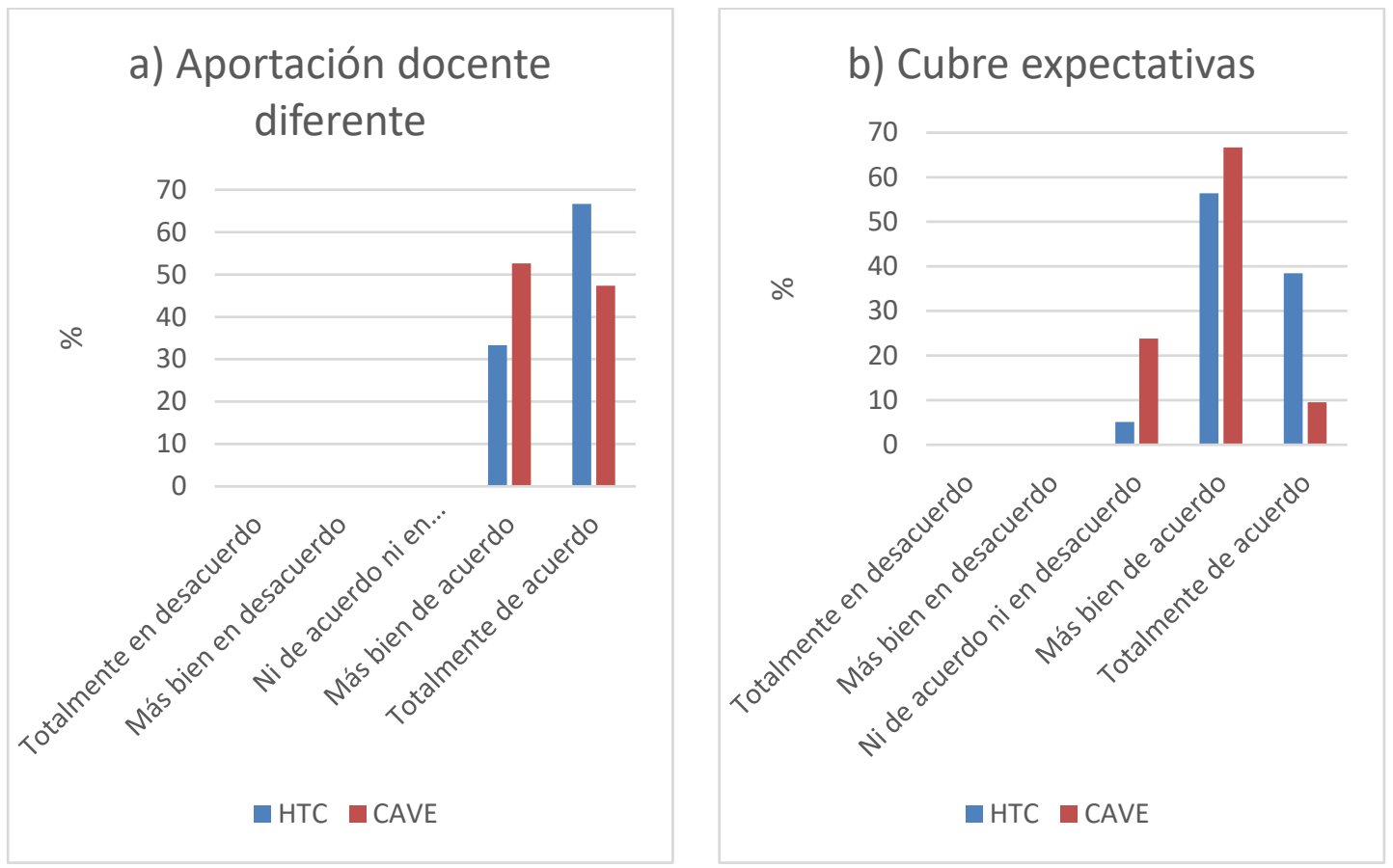

(c) ) BY-NC-ND 2020, Universitat Politècnica de València

Congreso In-Red (2020) 

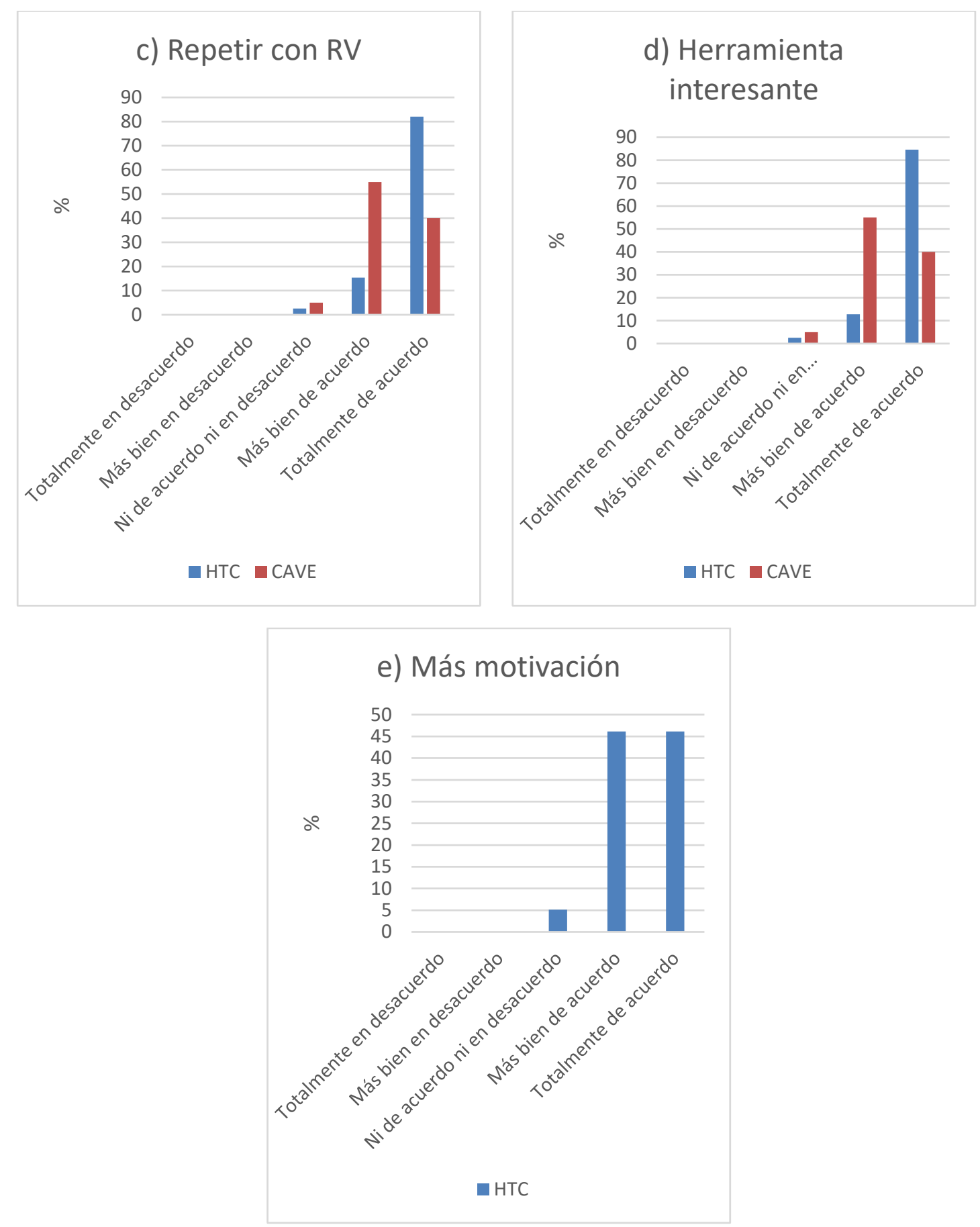

Fig. 11 Resultados de las encuestas a las preguntas:

a) Consideras que la herramienta ofrece una aportación distintiva con respecto a otras herramientas docentes vistas

b) La experiencia ha cubierto mis expectativas

c) Si tuviese la opción, me gustará volver a hacer uso de RV en futuras ocasiones

d) En su conjunto considero el uso de RV como una herramienta interesante en el diseño de productos

e) Considero que el uso de las gafas de RV me ha motivado a realizar un mejor diseño

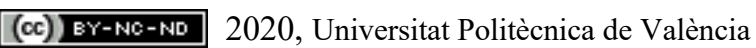

Congreso In-Red (2020) 
En la figura 11 se encuentran reflejados los resultados de cinco preguntas en formato Likert en las cuales se evalúa el impacto que han tenido estas tecnologías a nivel educativo y de satisfacción del alumnado. Cabe destacar que los resultados son todos en los niveles neutrales y positivos especialmente en el caso de las gafas HTC. De hecho, al igual que en las otras preguntas, los resultados han aumentado significativamente con el uso de este nuevo sistema respecto a la utilización del CAVE. La pregunta e es una nueva pregunta no realizada durante los años del CAVE y por eso no se disponen de datos, pero refleja que el alumnado se siente más motivado al utilizar herramientas RV y destinan más tiempo a obtener mejores resultados.

\subsection{Impacto a nivel laboral}

De los encuestados aproximadamente la mitad trabajan (21), con lo que se ha querido explorar la utilidad que tiene enseñar esta herramienta tecnológica para la perspectiva laboral. De acuerdo a los resultados vistos en la figura 12 se concluye que, aunque la mayoría de estos nuevos profesionales no la utiliza actualmente $(70 \%)$ a casi todos les gustaría utilizarla si estuviera dentro de sus posibilidades $(90 \%)$.

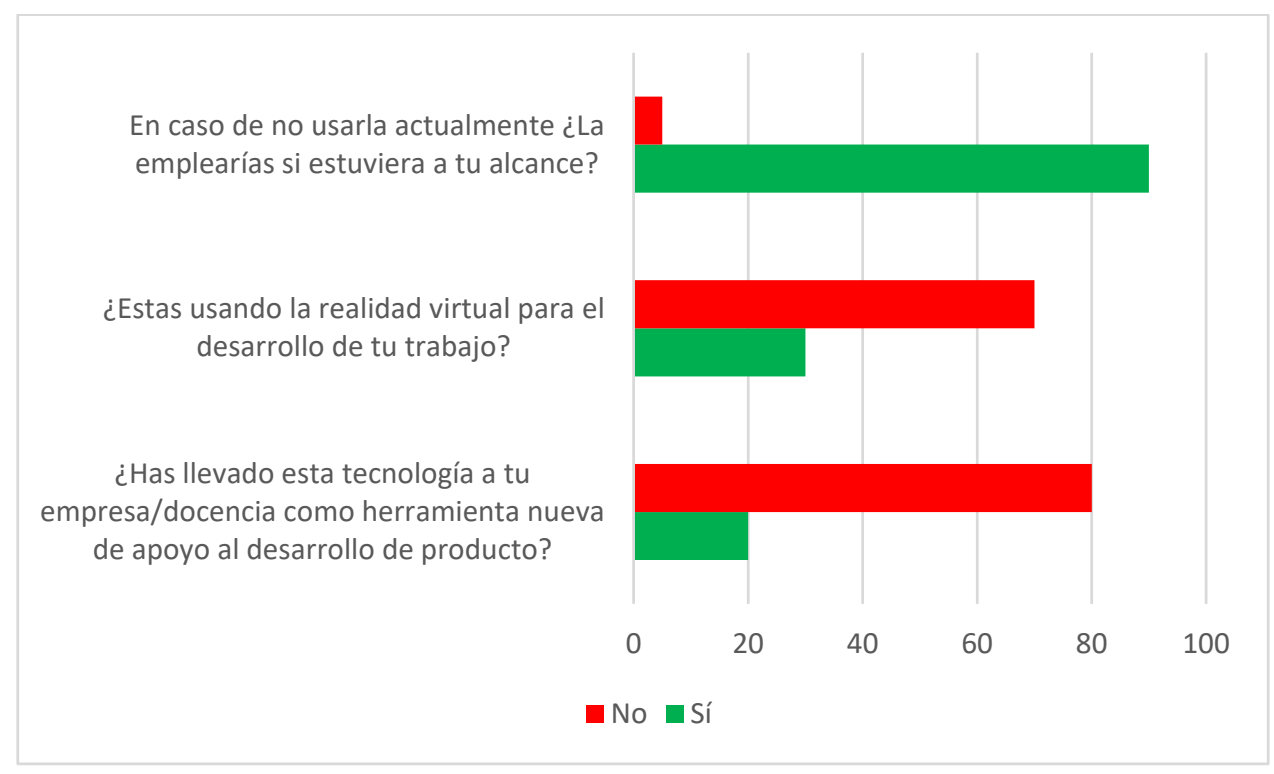

Fig. 12 Resultados de la encuesta respecto a la utilización de RV en el ámbito laboral 


\section{Conclusiones}

El empleo de técnicas que facilitan la simulación de espacios virtuales, la interactividad al detalle con los productos y la inmersión virtual del diseñador en su proceso de desarrollo, como sensación máxima de aproximación a la realidad, se ha configurado como una herramienta eficaz en el proceso de modelización y chequeo de nuevos productos que cada día está teniendo más presencia en el trabajo habitual del diseñador.

Además, a medida que la técnica avanza y la tecnología ofrece interfaces mejoradas, la relación que se establece entre el diseñador y el espacio inmersivo multiplica el proceso creativo, en calidad y experiencia, como así se muestra en la comparativa entre el uso de la CAVE y las HTC Vive en cuanto a la experiencia de inmersión, obteniendo una mejora ( $56 \%$ vs $32 \%$ ) en la validación de la escala e integración en su contexto (Fig.10).

La enseñanza de este tipo de herramientas al estudiante actual, caracterizado por ser nativo digital inmerso en la tecnología actual, resulta perfectamente alineado con sus inquietudes y motivaciones, valorándolas como innovadoras respecto a procesos de prototipado físicos tradicionales. En consecuencia, procesan una alta motivación en su aprendizaje (Fig. 11e) y aplicación, quedando cubiertas sus expectativas (Fig. 11b).

No solo eso, el tipo de alumnado actual como futuro profesional vive en un mundo de inmediatez y adaptación al cambio, por lo que el empleo de este tipo de tecnología, en sustitución de la fabricación de un prototipo físico, más alla de suponer un ahorro a medio plazo en cuanto a costes de material e inversión de tiempo, le resulta atractivo por las posibilidades que ofrece de modificación y adaptación inmediata del prototipo durante el proceso de trabajo y análisis del producto.

Por ello, la apuesta docente por el uso de estas tecnologías en la formación de los futuros diseñadores industriales parece imprescindible para estar, en mayor medida, preparados para el futuro mercado laboral. Esta tecnología estará, como ha sucedido históricamente, al alcande de la mayoría de los profesionales y empresas en el momento sus costes se reduzcan y nuestros egresados demuestren su utilidad y sentido de uso para el desarrollo del proyecto (Fig. 12). Ha sido precisamente la reducción de costes y la capacidad de nuestro dispositivos personales la que la ha acercado a nuestras aulas, creando un punto de no retorno, tal y como se demuestra en múltiples indicadores, como por ejemplo el aumento de un $40 \%$ en la intención de repetir con RV y de encontrar la herramienta motivadora en los procesos de desarrollo de producto (Fig. 11c).

\section{Agradecimientos}

Se agradece la colaboración y buen hacer del Área de Sistemas de la Información y Comunicaciones (ASIC) de la Universitat Politècnica de València (UPV), en particular a Fernando Serrano Carpena y a Manuel Jiménez López.

\section{Referencias}

BOGARD DOMINGO, J. (2015). Comparativa de métodos de visualización en soporte bidimensional y realidad virtual inmersiva en el proceso de diseño. Trabajo Final de Máster. Valencia: Universitat Politècnica de València.

BOOT, W., BLAKELY, D., SIMONS, D. (2011). "Do Action Video Games Improve Perception and Cognition?". Frontiers in psychology. Vol. 2. Article number 226, p. 1-7 
MOLINA, C.R. PERTEGAL, M. JIMENO, M.A. MORA, M.H. (2018). "Virtual Reality Learning Activities for Multimedia Students to Enhance Spatial Ability”. Sustainability, vol.10 (4), 1074.

RICHERT, A., SHEHADEH, M., WILLICKS, F., JESCHKE, S. (2016). Digital Transformation of Engineering Education. International Journal of Engineering Pedagogy, Volume 6, Issue 4, p.23-29.

RODRIGUEZ CHICOTE, A. (2012). Realidad virtual inmersiva y diseño asistido por ordenador aplicado al modelado de una cocina. Proyecto Final de Carrera. Valencia: Universitat Politècnica de València, $<$ http://riunet.upv.es/handle/10251/38714> [Consulta: 10 de marzo 2020]

RUBIO-TAMAYO, J.L., GÉRTRUDIX BARRIO, M., GARCÍA GARCÍA, F. (2016) "Virtuality continuum in communication and education: user experience and interaction design in simulation and interactivity approaches in real and virtual environments", 8th International Conference on Education and New Learning Technologies (EDULEARN), Editorial: EDULEARN Proceedings, Barcelona, p. 4526-4534.

SÁIZ MAUlEÓN, B., SANTONJA LlABATA, A., MUÑOZ VENDRELL. V. (2010). "Realidad Virtual: Aportaciones TIC a la docencia y sus aplicaciones en el desarrollo del proyecto ". En: XIII Congreso Internacional de Expresión Gráfica Arquitectónica. Universitat Politècnica de València. Editorial UPV. 4-4.

SALAH, B., HAIDER ABIDI, M., HAMMAD MIAN, S., KRID, M., ALKHALEFAH, H., ABDO, A. (2019). "Virtual Reality-Based Engineering Education to Enhance Manufacturing Sustainability in Industry 4.0", Sustainability, Vol. 11, p.1477.

VAL FIEL, M. (2015). “Entornos Virtuales para el Diseño de Producto en el Espacio Público". En: IN-RED. Congreso de Innovación Educativa y de Docencia en Red de la Universitat Politècnica de València. Editorial UPV. 1-13.

VERGARA, D., PABLO RUBIO, M., LORENZO, M. (2017). “On the Design of Virtual Reality Learning Environment in Engineering”. Multimodal Technologies and Interaction. Vol. 1, 11, P. 1-12. 